\title{
3-D Finite Element Investigation of Flux Regulation Performance of a Novel Hybrid Excitation Brushless Claw-Pole Alternator
}

\author{
Dongwei Qiao ${ }^{1,2, a}$, Yanhong $\mathrm{Li}^{2}$, Jianhua Shang ${ }^{2}$ and Hui Zhong ${ }^{3}$ \\ ${ }^{1}$ School of Control Science and Engineering, Shandong University, Jinan, China \\ 2 State Grid Taian Power Supply Company, Taian, China \\ ${ }^{3}$ Electrical Engineering School, Shandong University, Jinan ,250061 China
}

\begin{abstract}
In consideration of low power density of electric excitation claw-pole synchronous alternator (EECA) and some difficulties in magnetic field regulation of permanent magnet claw-pole synchronous alternator (PMCA), a novel hybrid excitation brushless claw-pole alternator (HEBCA) is proposed in this paper. Its structure and field control principle are described. Three dimensional finite element analysis is used to obtain the no-load magnetic field distributions and field control capability under different field currents. The result shows that the flux of the prototype machine can be adjusted over a wide range with a relatively low field current.
\end{abstract}

\section{INTRODUCTION}

The electric excitation claw-pole alternator (EECA) has been used widely in vehicle due to its simple structure, low cost, and easy to regulate. However, the existing of brushes and commutator significantly restrict their further develop-ment ${ }^{[1-3]}$.

The permanent magnet claw-pole alternator (PMCA) eliminates the field winding, brush and loop, and thus is simpler and more reliable. The efficiency and power density are improved as well since excitation is not required. However, the magnetic field's regulation is very challenging and hard to achieved ${ }^{[4,5]}$.

Hybrid excitation claw-pole alternators (HECA) have both permanent magnets (PMs) and field winding, and the two magnetizations sources are concurrent. Therefore, HECA takes the advantages of both the EECG and PMCG. Compared with traditional EECG, HECA has higher efficiency and power density, which makes it a very promising technique in recent years.

A novel hy-brid excitation brushless claw-pole alternator is proposed in this paper. The excita-tion winding is located on the excitation bracket fixed on the end closure of the al-ternator, thus brushes and slip rings are eliminated, and the reliability is greatly improved. Three-dimensional finite element analysis (FEA) is employed to calcu-late the magnetic field distributions.

\section{STRUCTURE}

Fig 1. shows the structure of the HEBCA, which consists mainly of stator, claw pole, PMs, excitation bracket and DC field windings. In this alternator, the conventional stator and three phase balanced armature windings are

\footnotetext{
a Corresponding author:283453643@qq.com
}

used here. There are two excitation brackets and field windings at the end of enclosures. The field winding joins with shield through this bracket. The tip and root of the claw pole have same width and thickness as shown in Fig2. Therefore, the brushes and slip-ring can be omitted. The cuboid permanent magnets are placed in the gaps between two claw poles as shown in Fig 3, The PMs have two functions, one is generating magnetic flux, and the other is reducing the leakage flux between the poles.

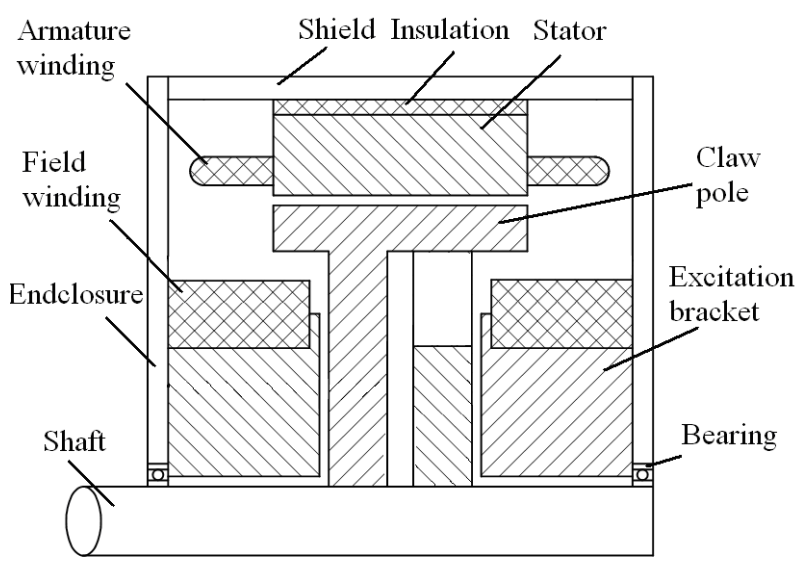

Fig 1. Structure of the novel HEBCA 


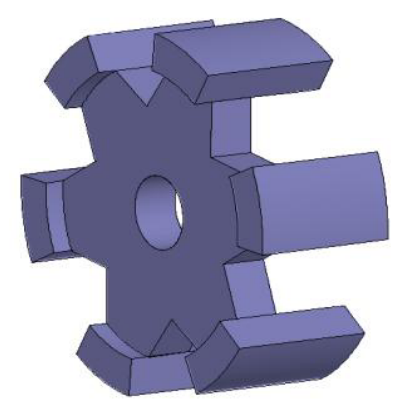

Fig 2. Claw pole

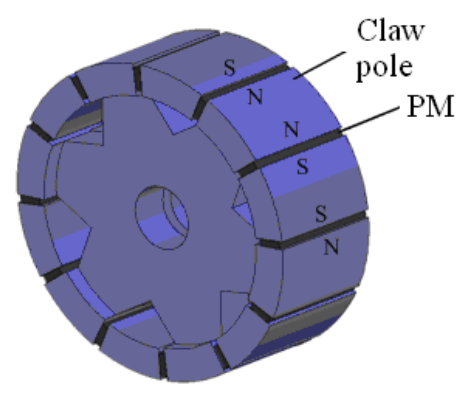

Fig 3. Claw pole with PMs

There are two magnetic sources in the HEBCA, i.e. the PMs and excitation current. The magnetic field is generated by both of them. When the excitation current is 0 , most of the magnetic flux generated by the PMs is closed in the rotor. Only a little gets into the stator, and the air-gap magnetic flux density is low.

When the excitation winding is energized, the magnetomotive force generated by PMs and excitation winding are in opposite direction, and the latter forces the former to get into the stator. The path of the magnetic flux generated by PMs is as follows: $\mathrm{PM} \rightarrow$ claw pole $\rightarrow$ main air-gap $\rightarrow$ teeth $\rightarrow$ stator yoke $\rightarrow$ teeth $\rightarrow$ main air-gap $\rightarrow$ claw pole $\rightarrow$ PM, while that of magnetic flux generated by excitation current is: excitation bracket $\rightarrow$ additional air-gap $\rightarrow$ claw pole $\rightarrow$ main air-gap $\rightarrow$ tooth $\rightarrow$ stator yoke $\rightarrow$ tooth $\rightarrow$ main air-gap $\rightarrow$ claw-pole $\rightarrow$ additional air-gap $\rightarrow$ excitation bracket. The two paths are in parallel and the air-gap magnetic flux increases with the excitation current.

\section{FEA-BASED INVESTIGATION OF MAGNETIC FIELD REGULATION}

In order to get the accurate magnetic field distribution and evaluate the field regulating performances of the HEBCA, 3D finite element analysis is performed on a model alternator. The detailed parameters for the model are shown in Table 1.
Table 1. Structure parameters of the HEBCA

\begin{tabular}{l|c}
\multicolumn{1}{c|}{ parameters } & values \\
\hline Additional Air-gap length $/ \mathrm{mm}$ & 0.2 \\
Number of poles & 12 \\
Outer diameter of stator core $/ \mathrm{mm}$ & 198 \\
Inner diameter of stator core $/ \mathrm{mm}$ & 152 \\
Length of stack $/ \mathrm{mm}$ & 55 \\
Air-gap length $/ \mathrm{mm}$ & 0.5 \\
Additional air-gap length $/ \mathrm{mm}$ & 0.2 \\
Width of pole $/ \mathrm{mm}$ & 35.5 \\
Thickness of pole $/ \mathrm{mm}$ & 15.5 \\
Diameter of rotor yoke $/ \mathrm{mm}$ & 80 \\
Height of PM $/ \mathrm{mm}$ & 15 \\
Thickness of PM/mm & 8 \\
Length of PM/mm & 18 \\
\hline
\end{tabular}

To reduce the computation time, the FEA is performed on the domain covering two poles, as shown in Fig 4. while Fig 5. shows the mesh of HEBCA model.

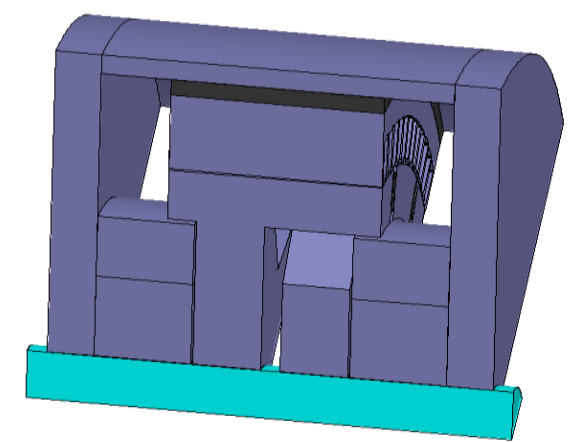

Fig 4. FEA model of the HEBCA

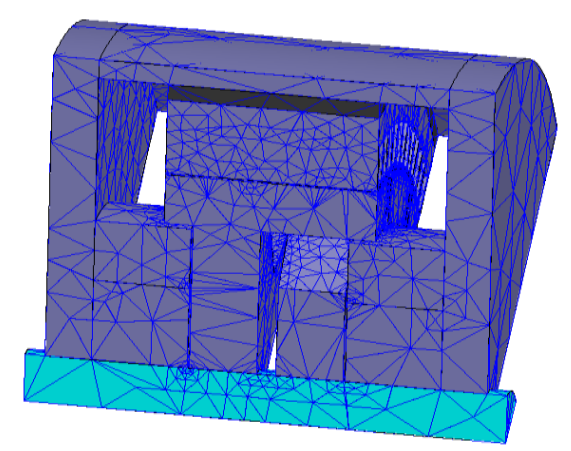

Fig 5. Grid mesh of the HEBCA

Based on the above model, the no-load magnetic field of the HEBCA at different field currents (labeled as $I_{f}$ ) could be obtained, and the distributions of the air-gap flux density are shown in Fig 6. 


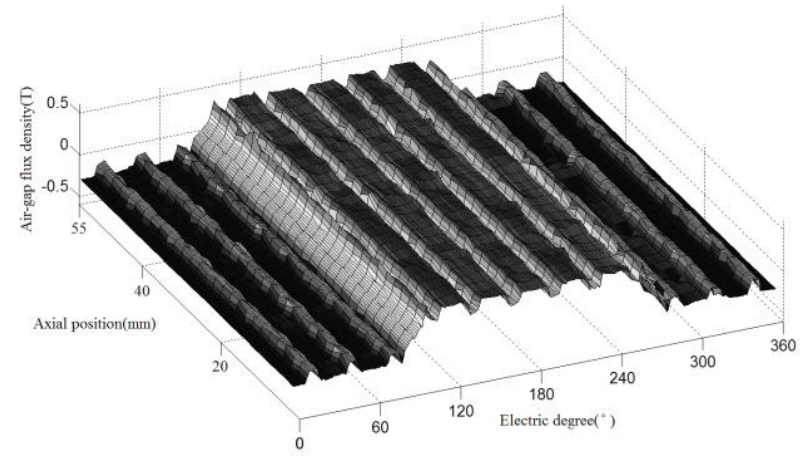

(a) $\mathrm{I}_{\mathrm{f}}=0$

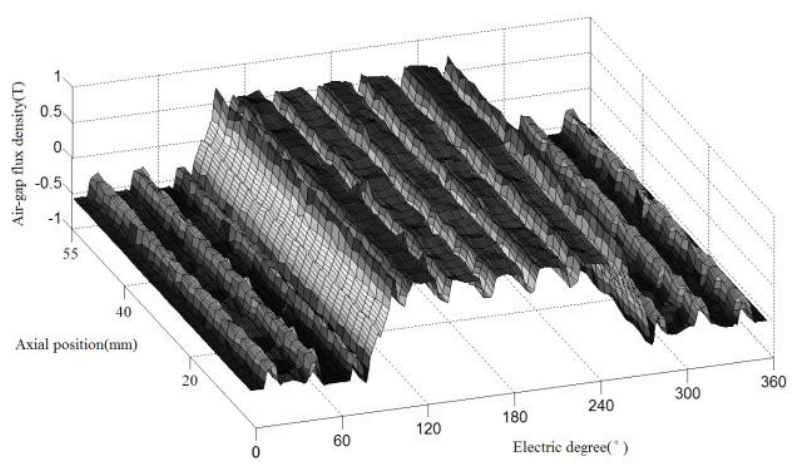

(b) $\mathrm{I}_{\mathrm{f}}=5 \mathrm{~A}$

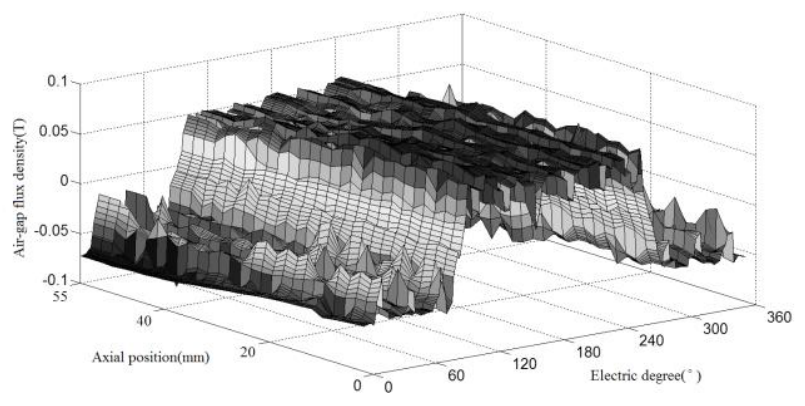

(c) $\mathrm{I}_{\mathrm{f}}=-5 \mathrm{~A}$

Fig 6. Air-pap flux density distributions at different field currents

Figure 7. shows the ability of the magnetic field adjustment of the alternator. The following conclusions could be get:

(1) When the excitation current is $0 \mathrm{~A}$, the air gap of the electric excitation claw pole alternator is 0T, while the average magnetic density of the hybrid excitation claw pole alternator is $0.18 \mathrm{~T}$.

(2) When the excitation current is positive, the air gap flux density of the HEBCA is more than the EECA's air gap flux density. With the excitation current increasing, the maximum value of average air gap flux density of HEBCA is $0.51 \mathrm{~T}$, while the maximum value of average air gap flux density of EECA is 0.41T. Compared with the EECA, the maximum value is increased by $24.4 \%$.

(3) When the excitation current in the range from 0 to $10 \mathrm{~A}$, the excitation current of the HEBCA is less than the EECA's. So, the HEBCA has the less excitation loss.

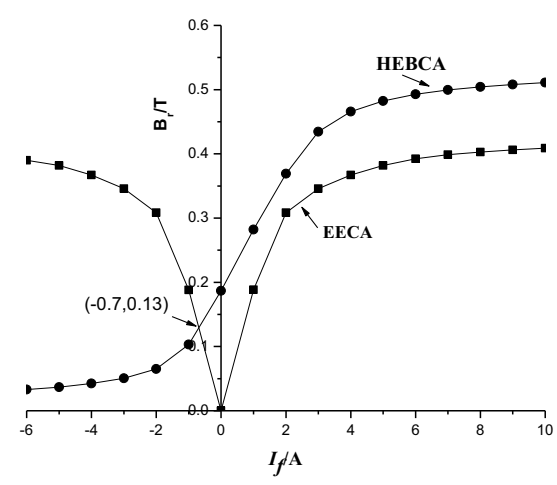

Figure 7. Average air-gap flux density for different field currents.

\section{Conclusion}

A novel hybrid excitation brushless claw-pole alternator is proposed in this paper. The structure and magnetic field regulation principle are introduced. The no-load magnetic field of the alternator is calculated by 3-D finite element analysis. The following conclusions can be summed up:

(1) The structure of this hybrid excitation reduces the alternator's leakage flux, improves the airgap flux density. Compared with the electric excitation brushless claw-pole alternator, the HEBCA has a higher air-gap flux density and power density.

(2) The hybrid excitation brushless claw-pole alternator generates the same air gap flux density with a smaller excitation current than the electric excitation claw-pole alternator, thus reduces the excitation loss and improves the efficiency of the alternator.

(3) The excitation winding is fixed on the excitation bracket, thus brushes and slip-ring is eliminated and the operation reliability is improved.

\section{Acknowledgments}

The work has been supported by National Natural Science Foundation of China (NSFC) under grant \#51307100.

\section{References}

1. Christian Kaehler, Gerhard Henneberger. Eddycurrent computation in the claws of a synchronous claw-pole alternator in generator mode[J]. IEEE Transactions on Magnetics, 38(2): 1201-1204,(2002).

2. Christian Kaehler, Gerhard Henneberger. Transient 3-D FEM computation of eddy-current losses in the rotor of a claw-pole alternator[J]. IEEE Transactions on Magnetics, 40(2): 1362-1365, (2004). 
3. Ramesohl I, Henneberger G, Kuppers S, etc. Three dimensional calculation of magnetic forces and displacements of a claw-pole generator[J]. IEEE Transactions on Magnetics, 32(3): 1685-1688, (1996).

4. Wang R, Demerdash N A. Computation of load performance and other parameters of extra high speed modified lundell alternators from 3D-FE magnetic field solutions[J]. IEEE Transactions on Energy Conversions, 7(2): 342-352,(1992).

5. Hua Bai, Steven D. Pekarek, Jerry Ticheoner, etc. Analytical derivation of a coupled-circuit model of a claw-pole alternator with concentrated stator windings $[\mathrm{J}]$. IEEE Trans. on Energy Conversions, vol.17, no.1, pp. 32-38, (2002). 